\title{
Hybrid Photonic Crystal Lasers
}

\author{
Alexandros A. Liles ${ }^{1}$, Andrei P. Bakoz ${ }^{2,3}$, Alfredo A. Gonzalez ${ }^{1}$, Tatiana Habruseva ${ }^{2}$, Saydulla \\ Persheyev $^{1}$, Guillaume Huyet ${ }^{2,3}$, Stephen P. Hegarty ${ }^{2,3}$, Liam O'Faolain ${ }^{1,2,3}$ \\ ${ }^{1}$ Scottish Universities Physics Alliance (SUPA), School of Physics and Astronomy, St Andrews, UK \\ ${ }^{2}$ Tyndall National Institute, Cork, Ireland \\ ${ }^{3}$ Cork Institute of Technology, Cork, Ireland \\ ${ }^{1}$ SUPA, School of Physics and Astronomy, University of St. Andrews, UK \\ ${ }^{2}$ Tyndall National Institute, Cork, Ireland \\ ${ }^{3}$ Centre for Advanced Photonics and Process Analysis, Cork Institute of Technology, Cork, Ireland \\ liam.ofaolain@tyndall.ie
}

\begin{abstract}
Energy efficient Wavelength Division Multiplexing (WDM) is the key to satisfying the future bandwidth requirements of datacentres. As the silicon photonics platform is regarded the only technology able to meet the required power and cost efficiency levels, the development of silicon photonics compatible narrow linewidth lasers is now crucial. We discuss the requirements for such laser systems and report the experimental demonstration of a compact uncooled external-cavity mWatt-class laser architecture with a tunable Si Photonic Crystal resonant reflector, suitable for direct Frequency Modulation.
\end{abstract}

Keywords: silicon photonics, photonic crystal, resonant mirror, semiconductor laser

\section{INTRODUCTION}

The large scale movement and storage of data has become an essential component of society. A significant fraction of the world's economy is now based on the internet and is an important area for future growth. Achieving efficient data manipulation and communication within datacentres is a key challenge for the 21 st century and is a prerequisite for providing billions of people with the means to access, move and manipulate the huge volumes of information they are now creating. Only with ground breaking rates of data communication can the expected levels of performance be achieved in delivering both current and next generation information based systems. Optical interconnections are the only means to provide the required communications bandwidth. However, current solutions to optical interconnects are still orders of magnitude away from the efficiencies (cost, power and volume) that are and increasingly will be required to assure reliable, fast, low cost and energy efficient data centres.

Silicon Photonics is one of the most promising technologies for cost-effective fabrication of photonic components and integrated circuits, however, there are some major challenges in terms of device performance. The development of efficient light emission from silicon has been the subject of a large body of research [1-3] but still remains elusive. The practical solution is the heterogeneous integration of III-V component on the silicon based photonic integrated circuits by means of direct wafer bonding [4], or the hybrid integration of III-Vs by means of flip-chip bonding [5].

Electro-optic modulators are then the next key component. The electrical data stream, consisting of a time varying voltage, (supplied by some external source) is typically converted into a time varying optical intensity for transmission across the data interconnect. The application of an electric field to a material can result in changes to the real and imaginary parts of the refractive index. The Pockels effect, the Kerr effect and the Franz-Keldysh effect are traditionally used in semiconductor materials to realise modulation based on either electro-refractive or electro-absorption modulation, however, in pure Silicon (the preferred material for mass production, at the telecommunications wavelengths) these are very weak. With thermo-optic effects too slow for useful datacommunication [6], the plasma dispersion (change in concentration of electrons) effect [7] has become the preferred mechanism for high speed modulation of optical signals with silicon photonics. In conventional approaches, this 
change in refractive index is translated into intensity modulation by means of a Mach-Zehnder Interferometer (MZI). Due to the relatively small modification in refractive index possible [8] long devices are invariably required to realise modulation. As the capacitance of a silicon device is approximately $1 \mathrm{fF} / \mu \mathrm{m}^{2}$, a $1.5 \mathrm{~mm}$ long MZI modulator with $2 \mathrm{~V}$ voltage swing, has a power consumption of $\sim 5 \mathrm{pJ} / \mathrm{bit}$. The use of advanced waveguide-pn junction designs can be used to enhance the change in refractive index and while this reduces the length to $0.5 \mathrm{~mm}$, the power consumption is unchanged. Hence Silicon based modulators suffer from both large size and more importantly the high capacitance which consumes energy (and generates heat) in high data rate communications.

Resonant modulators $[9,10]$ offer a solution to the problems of large devices with high power consumption. In contrast to a single-pass device, at the resonant wavelength light makes many round trips in the resonator experiencing the small refractive index change many times (proportional to the finesse of the cavity). Consequently, the change in the total number of carriers required to change the optical transmission is orders of magnitude less than that needed in MZI-modulators, with similar reduction in footprint. The RF power required to drive these electrons in and out of the device is consequently reduced. The need for travelling wave electrodes is avoided as the device may be treated as a lumped element, dramatically reducing the complexity, power consumption and cost of the driver circuit (typically greater than the modulator power consumption in the MZI case).

Slowlight photonic crystal offer one solution to this problem. Traditionally losses have been a major drawback, however, recent developments have largely overcome this issue [11,12] and have enabled a ten times reduction in length [13]. Photonic Crystal cavities based modulators take this to the extreme, providing large reductions in footprint and power consumption relative to ring resonators $[14,15]$, see Figure 1. But the resonant nature of such a modulator makes it intrinsically wavelength selective, creating matching issues between it and the external laser which requires the use of tuning elements and control circuitry [16]. The ensuing cost in terms of power and complexity has made the broadband MZI the preferred solution.

\section{PHOTONIC CRYSTAL LASER}

In this work, we report a compact hybrid external cavity laser architecture, appropriate for direct modulation by frequency tuning. The laser cavity is formed by butt-coupling a short Reflective Semiconductor Optical Amplifier (RSOA) to SOI chips containing tunable Photonic Crystal (PhC) cavity-based resonant reflectors, similar to those of [14], providing a route to accessing the advantages of resonant modulation without the wavelength matching issues. PhC cavities provide very precise control of their resonant wavelengths by lithographic tuning [17], significantly assists channel scalability. Hybrid integration provides a means to combine the III-V and silicon components. Flipchip bonded versions of these lasers [18] are suitable for integration with our previously demonstrated active and passive $\mathrm{PhC}$ components for the realization of more complex power-efficient Si photonic systems 


\section{Mach Zehnder Interferometer}

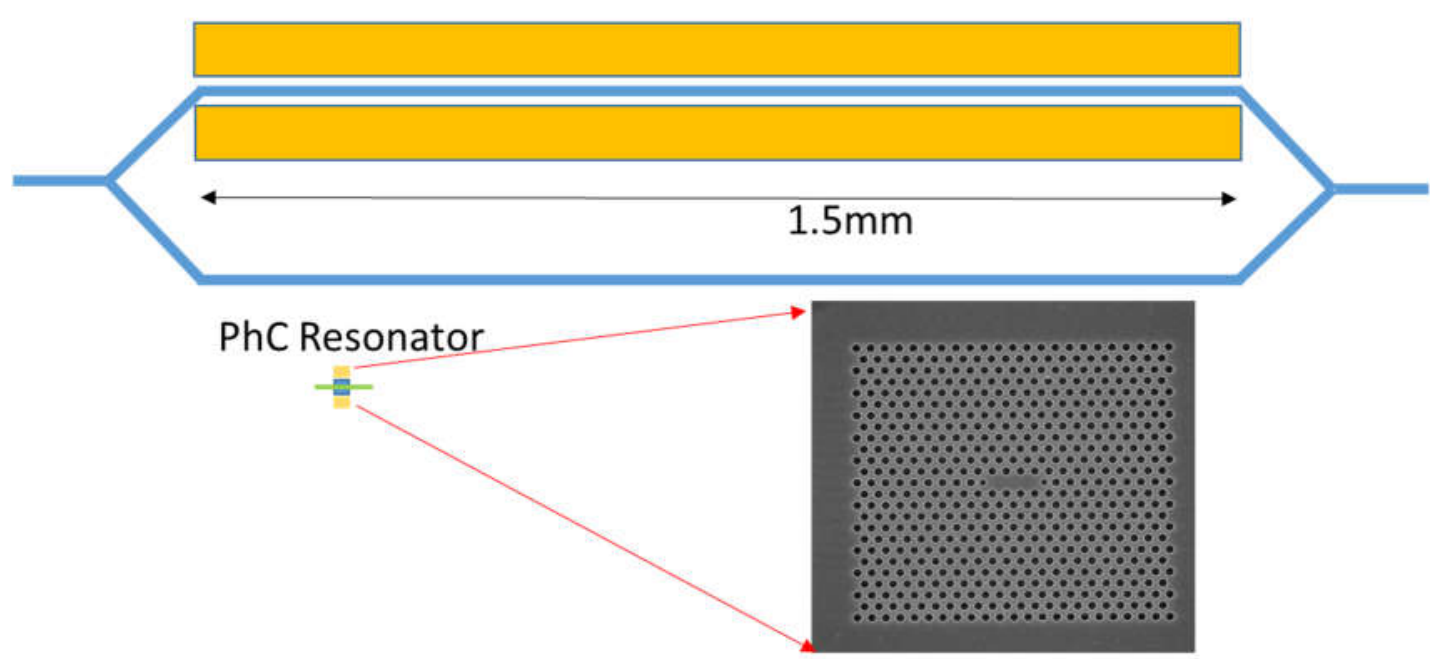

Figure 1 Schematic of an MZI and PhC resonator drawn on the same scale (the PhC resonator is also shown magnified). Patterned Silicon is shown in blue and metal contacts in gold

A schematic of the proposed laser is presented in Fig. 1. The RSOA chip was a $250-\mu \mathrm{m}-$ long InPbased amplifier with HR coated back facet and AR coated front facet, bonded to a ceramic tile/carrier. The Si passive chip was fabricated on a 220-nm SOI platform and comprised an SU8 polymer bus waveguide vertically coupled to a Dispersion Adapted (DA) PhC cavity, with a p-i-n junction extending in the PhC defect as described in [14]. The physical principle behind the functionality of the latter component as a resonant reflector is described in $[17,19]$. The bus waveguide height and width were $\sim 2.1 \mu \mathrm{m}$ and $\sim 3.5 \mu \mathrm{m}$, respectively for better matching with the emitted mode of the RSOA. Both facets of the Si chip were AR coated to suppress parasitic reflections. The lasing emission is a result of the frequency matching of the longitudinal modes of the laser cavity with the reflection peaks of the PhC cavity at resonance. The short laser cavity length ensures that only one longitudinal mode overlaps with each reflection. Due to their narrow linewidths, the reflections vary rapidly over a few $\mathrm{GHz}$, and thus the electro-optical modulation of the $\mathrm{PhC}$ cavity resonances results will result in a change in the phase matching condition and consequently a shift of the frequency of the overlapping longitudinal mode. The threshold currents were as low as $9 \mathrm{~mA}$ in the and over a significant current range (typically for up to $65 \mathrm{~mA}$ ), single mode operation with laser linewidths less than $4.5 \mathrm{MHz}$ was achieved. The gain chip is based a ridge waveguide structure with AlInGaP quantum wells and shows very good performance at elevated temperatures. All measurements shown here were made without active cooling.
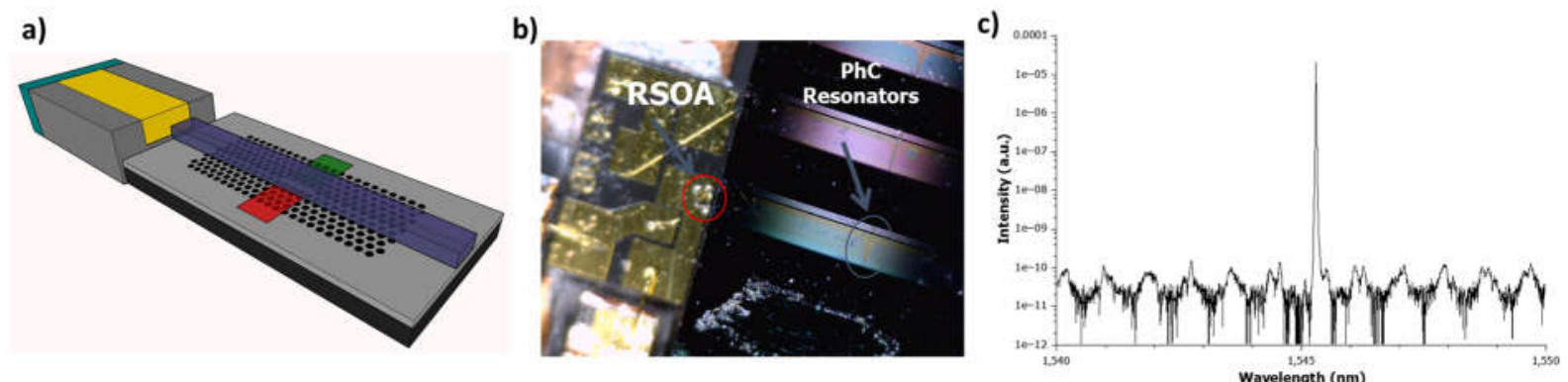

Figure 2 a) A schematic of the hybrid laser b) An optical microscope image showing the gain chip buttcoupled to the silicon chip c) Example lasing spectrum showing high side mode suppression. 


\section{CONCLUSIONS}

We have demonstrated a compact, external-cavity, hybrid III-V/Si laser architecture, comprising an RSOA butt-coupled to a silicon chip with $\mathrm{PhC}$ cavity-based that acts a resonnt mirror. Output optical powers up to $3 \mathrm{~mW}$, with wcWPE up to $4.2 \%$ and SMSRs up to $55 \mathrm{~dB}$ were observed. Since PhC cavities show extremely small footprint and wide free spectral range as a result of their high $\mathrm{Q} / \mathrm{V}$ ratio, the architecture here presented an ideal candidate for WDM applications, especially in conjunction with other PhC cavity solutions. This configuration gives automatic matching of the resonant modulation and the laser wavelength and provides a route for the use of silicon nano-resonators in practical optical links.

\section{ACKNOWLEDGEMENTS}

We acknowledge funding from the European Research Council (Starting Grant 337508) and the Engineering and Physical Sciences Research Council (Doctoral Grant EP/L505079/1 and Equipment Grant EP/L017008/1)

\section{REFERENCES}

[1] M. Grydlik, F. Hackl, H. Groiss, M. Glaser, A. Halilovic, T. Fromherz, W. Jantsch, F. Schaeffler \& M. Brehm "Lasing from Glassy Ge Quantum Dots in Crystalline Si," ACS Photonics 3, 298-303 (2016);

[2] R. L. Savio, S. L. Portalupi, D. Gerace, A. Shakoor, T. F. Krauss, L. O'Faolain, L. C. Andreani \& M. Galli "Room-temperature emission at telecom wavelengths from silicon photonic crystal nanocavities," Applied Physics Letters 98, 201106 (2011)

[3] L. Pavesi, L. D. Negro, C. Mazzoleni, G. Franzo \& F. Priolo "Optical gain in silicon nanocrystals," Nature 408, 440-440 (2000)

[4] G. Roelkens, L. Liu, D. Liang, R. Jones, A. Fang, B. Koch, and J. Bowers, Laser Photonics Rev. 4, 751 (2010).

[5] S. Tanaka, S.-H. Jeong, S. Sekiguchi, Teruo, Kurahashi, Y. Tanaka \& K. Morito "High-output-power, single-wavelength silicon hybrid laser using precise flip-chip bonding technology," Optics Express 20, 28057-28069 (2012)

[6] D. M. Beggs, T. P. White, L. Cairns, L. O'Faolain \& T. F. Krauss "Ultrashort Photonic Crystal Optical Switch Actuated by a Microheater," IEEE Photonics Technology Letters 21, 24-26 (2009)

[7] A. Liu, R. Jones, L. Liao, D. Samara-Rubio, D. Rubin, O. Cohen, R. Nicolaescu \& M. Paniccia "A high speed silicon optical modulator based on a metal-oxide-semiconductor capacitor," Nature 427, 615-618 (2004)

[8] R. Soref \& B. Bennett "Electrooptical effects in silicon," Quantum Electronics, IEEE Journal of 23, 123$129(1987)$

[9] Q. Xu, B. Schmidt, S. Pradhan \& M. Lipson "Micrometre-scale silicon electro-optic modulator," Nature 435, 325-327 (2005)

[10] F. Gardes, A. Brimont, P. Sanchis, G. Rasigade, D. Marris-Morini, L. O'Faolain, F. Dong, J. Fedeli, P. Dumon, L. Vivien, T. Krauss, G. Reed \& J. Marti "High-speed modulation of a compact silicon ring resonator based on a reverse-biased pn diode," Optics Express 17, 21986-21991 (2009)

[11] L. O'Faolain, S. A. Schulz, D. M. Beggs, T. P.White, M. Spasenovic, L. Kuipers, F. Morichetti, A. Melloni, S. Mazoyer, J. P. Hugonin, P. Lalanne \& T. Krauss "Loss Engineered Slow light Waveguides," Optics Express 18, 27627-27638 (2010)

[12] H. S. Dutta, A. K. Goyal, V. Srivastava \& S. Pal "Coupling light in photonic crystal waveguides: A review," Photonics and Nanostructures - Fundamentals and Applications 20, 41 - 58 (2016)

[13] H. C. Nguyen, N. Yazawa, S. Hashimoto, S. Otsuka \& T. Baba "Sub-100 \$m photonic crystal Si optical modulators: spectral, athermal, and high-speed performance," Selected Topics in Quantum Electronics, IEEE Journal of 19, 127-137 (2013)

[14] K. Debnath, L. O'Faolain, F. Y. Gardes, A. G. Steffan, G. T. Reed \& T. F. Krauss "Cascaded modulator architecture for WDM applications," Optics Express 20, 27420-27428 (2012)

[15] S. Meister, H. Rhee, A. Al-Saadi, B. Franke, S. Kupijai, C. Theiss, H. Eichler, D. Stolarek, H. Richter, L. Zimmermann, B. Tillack, M. Lesny, C. Meuer, C. Schubert \& U. Woggon "High-Speed Fabry Optical Modulator in Silicon With 3um Diode," Lightwave Technology, Journal of 33, 878-881 (2015) 
[16] X. Zheng, Y. Luo, J. Lexau, F. Liu, G. Li, H. Thacker, I. Shubin, J. Yao, R. Ho, J. Cunningham \& A. Krishnamoorthy "2-pJ/bit (On-Chip) 10-Gb/s Digital CMOS Silicon Photonic Link," Photonics Technology Letters, IEEE Journal of 24, 1260-1262 (2012)

[17] A. A. Liles, K. Debnath \& L. O'Faolain "Lithographic wavelength control of an external cavity laser with a silicon photonic crystal cavity-based resonant reflector," Optics Letters 41, 894-897 (2016)

[18] S. Tanaka, S.-H. Jeong, S. Sekiguchi, Teruo, Kurahashi, Y. Tanaka \& K. Morito, "High-output-power, single-wavelength silicon hybrid laser using precise flip-chip bonding technology," Optics Express 20, 28057-28069 (2012)

[19] Y. De Koninck, G. Roelkens \& R. Baets "Design of a Hybrid III-V-on-Silicon Microlaser With Resonant Cavity Mirrors," Photonics Journal, IEEE 5, 2700413 (2013) 